\title{
Direct Method of the Minimum-Time Traversing and Hoisting Motion of the Container Crane
}

\author{
Subchan $^{1}$
}

\begin{abstract}
In this paper, a dynamic model of the container crane which represents simultaneous traversing and hoisting motions is discussed. The model is derived using Lagrangian modeling techniques. The problem is to minimize the transfer time of a container, where the optimal trajectory should satisfy the specified initial and terminal conditions and some constraints. The optimal control problem is transformed into sequence of nonlinear constrained optimization problems by discretising of the state and/or control variables. Numerical examples are provided, including the case where there is a singular-arc on control.
\end{abstract}

Keywords-Container crane, Nonlinear optimal control, Singular control, Direct method.

\section{INTRODUCTION}

$\mathrm{C}$ ontainer cranes are widely used to handle loading and unloading process of the containers at harbour. The fundamental motions are travelling of the trolley and hoisting of the cable. When the vessel is unloaded, the container is first transferred from the ship to the waiting truck. The truck then carries the container to the open storage area. When the ship is loaded the same problem is encountered.

The globalization has significantly increased the demand for logistics and transportation, particularly the demand for containerized vessel shipping. Due to the increasing volume of world-wide container traffic, container terminals have become more important component of global logistics networks. Container terminals serve as hubs for the transshipment of containers from ships to ships or other modes of transportation (i.e. rail and trucks). Therefore, container handling at harbour is very important and has to be minimized economically, especially the service time of the container vessel at the harbour. The general transfer of the container can be broken down into three parts: (i) vertical lifting, (ii) transfer of the container from starting point to the desired ending point, and (iii) lowering of the container. The transfer for parts (i) and (iii) require only the hoisting control of the crane. The part (ii) of the transfer requires both the hoisting and traverse controls and has the greatest potential for reduction of the overall transfer time.

The focus of this paper is to determine an optimal time trajectory of transferring the container from a given initial

\footnotetext{
Manuscript received May 1, 2007; revised November 1, 2007

Subchan is with Department of Mathematics, FMIPA, Institut Teknologi Sepuluh Nopember, Surabaya, INDONESIA. (email: Subchan@dsl.pipex.com)
}

position at rest to a given terminal position where it is required at rest again whilst satisfying some constraints. The container crane has fixed position with respect to the ship when loading and unloading processes are performed. Thus the whole processes are accomplished by a planar motion of the container hanging at the moving trolley. There are some constraints which are the finiteness of the actuators, safety regulations, trolley speed, hoisting speed, force of the trolley drive system and force of the hoisting drive system.

This paper is organized as follows. The dynamics model of container crane is described in Section 2. The formulation of the container crane as optimal control problem is presented in Section 3. Section 4 introduces the optimal control theory. The parameterization of the optimal control problem using a direct collocation approach is discussed in Section 5. Results of the numerical results and related numerical issues are given in Section 6. Finally, this paper closes by some conclusions in Section 7 .

\section{Modeling of The Container CRAnE}

The container crane is modelled as a two-dimensional system. The crane has a fixed position with respect to the ship. The elastic deformability of the crane construction and the cable will be neglected. It is assumed that all elements are infinite stiffness and there is no friction. We derive the dynamic equation for the system using the Lagrangian modelling techniques.

The container crane is modelled as system with $3 \mathrm{deg}$ rees of freedom. The model has two control inputs, which are force acting on the trolley in horizontal direction and the hoisting cable force. The mass of the container and the mass of trolley are and respectively. The model can be given as follows:

$$
\ddot{z}=\frac{F_{1}\left(m_{c}+G_{h}\right)+F_{h} m_{c} \sin \theta+m_{c} g \sin \theta \cos \theta G_{h}+m_{c} l \dot{\theta}^{2} \sin \theta G_{h}}{\left(m_{c}+G_{h}\right)\left(m_{t}+G_{t}\right)+m_{c} G_{h}\left(1-\cos ^{2} \theta\right)}
$$

$\ddot{\theta}=\frac{2 i \dot{\theta}+g \sin \theta+\dot{x} \cos _{2} \theta}{l}$

$i=\frac{-F_{h}+m_{c} l \dot{\theta}^{2}+m_{c} g \cos \theta G_{h}-m_{c} \ddot{z} \sin \theta}{m_{c}+G_{h}}$

Where $\mathrm{z}$ is the trolley position, $\dot{z}$ is speed of the trolley. $\theta$ is swing angle of the cable, $\dot{\theta}$ is swing angle speed, $l$ is length of the cable, $i$ is the hoisting speed, $G_{h}$ and $G_{t}$ are the virtual hoisting motor mass and the virtual trolley motor mass respectively. 


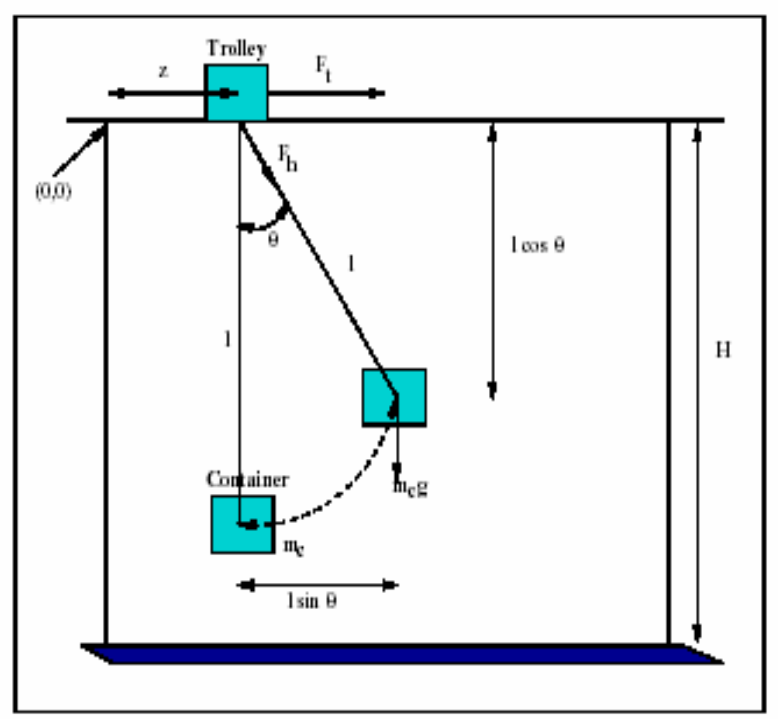

Figure 1. Schematic drawing of the container cranes

\section{PROBLEM Formulation}

The problem is to find the trajectory of the container cranes from an assigned initial state to a final state with the minimum time. This problem can be formulated by introducing the performance criterion

$\min \quad J=\int_{t_{0}}^{t_{f}} d t$

The performance criterion (2) is subject to the equations of motion (1). The equations (1) can be rewritten as first order differential equations as follows:

$\dot{x}_{1}=x_{2}$

$\dot{x}_{2}=\frac{F_{1}\left(m_{c}+G_{h}\right)+F_{h} m_{c} \sin x_{3}+m_{c} g \sin x_{3} \cos x_{3} G_{h}+m_{c} x_{5} x_{2}^{4} \sin x_{3} G_{h}}{\left(m_{c}+G_{h}\right)\left(m_{t}+G_{t}\right)+m_{c} g_{h}\left(1-\cos ^{2} x^{3}\right)}$

$\dot{x}_{3}=x_{4}$

$x_{4}=-\frac{2 x_{6} x_{4}+g \sin x_{3}+\dot{x}_{2} \cos x_{3}}{x_{5}}$

$\dot{x}_{5}=x_{6}$

$\dot{x}_{6}=\frac{-F_{h}+m_{c} x_{5} x_{4}^{2}+m_{c} g \cos x_{3} G_{h}-m_{c} \dot{x}_{2} \sin x_{3}}{m_{c}+G_{h}}$

Where

$x_{1}=\left\{x_{1},=z, x_{2}=\dot{z}, x_{3}=\theta, x_{4}=\dot{\theta}, x_{5}=l, x_{6}=i\right\}$

In addition, some constraints are described as follows: Initial and final conditions:

$x_{0}=\left\{0,0,0,0, l_{0}, 0\right\}$

$x_{t_{f}}=\left\{z_{t_{f}}, 0,0,0, l_{t_{f}}, 0\right\}$

Control constraints:

$$
\begin{aligned}
& F_{t_{\min }} \leq F_{t} \leq F_{t_{\max }} \\
& 0 \leq F_{h} \leq F_{h_{\max }}
\end{aligned}
$$

State constraints

$-5 \leq x_{2} \leq 5$

$-0,2 \leq x_{3} \leq 0.2$

$-5 \leq x_{4} \leq 5$

$-5 \leq x_{6} \leq 5$

\section{OPTIMAL CONTROL THEORY}

The problem is to find an admissible control, which minimises the performance index:

$\min J=\Phi\left[x\left(t_{f}\right), t_{f}\right]+\int_{t_{0}}^{t_{f}} L[x(t), u(t), t] d t$

with respect to the state vector functions:

$X=\left\{x:\left[o, t_{f}\right] \rightarrow \Re^{n} \mid \begin{array}{l}x_{i}, i=1, \ldots, n, \\ \text { piecewise continously differentiable }\end{array}\right\}$

and the control vector functions:

$U=\left\{u:\left[0, t_{f}\right] \rightarrow U \subset \mathfrak{R}^{n} \mid u_{i}, i=1, \ldots . ., n\right\}$ piecewise

continous

Subject to the following constraints:

$\dot{x}=f(x(t), u(t)) f: \Re^{n+m} \rightarrow \Re^{n}$

$x(0)=x_{0} \in \mathfrak{R}^{n} x_{0}$ known

$\Psi\left(x\left(t_{f}\right), t_{f}\right)=0 \in \mathfrak{R}^{p} \quad \Psi: \Re^{n} x \mathfrak{R}_{+} \rightarrow \mathfrak{R}^{p}$

$\mathrm{p} \leq \mathrm{n}, \mathrm{t}_{\mathrm{f}}$ unknown

$C(x(t), u(t)) \leq 0 \in \Re^{q}$

$C: \mathfrak{R}^{n+m} \rightarrow \mathfrak{R}^{q}$

$S(x(t)) \leq 0 \in \Re^{s}$

$S: \mathfrak{R}^{n} \rightarrow \mathfrak{R}^{s}$

The performance index describes a quantitative measure of the performance of the system over time. Here $\Phi: \Re^{n+1} \rightarrow \mathfrak{R}^{1}$ and $L: \Re^{n+m} \rightarrow \mathfrak{R}^{1}$ assumed to be sufficiently often continuously differentiable in all arguments.

Minimising $\mathrm{J}$ with respect to the control function $u$ must be accomplished in a way consistent with the dynamics of the system, whose performance is optimised. In other words, equation (15) is the first fundamental equality constraint. The optimal control $U^{*}$, when substituted to (15), will produce the optimal state $X^{*}$, while minimising $J$.

The optimal state $X^{*}$ is further constrained by the boundary conditions (16) and (17), path constraints (18) and (19). Finally, it should be mentioned that (18) or (19) may be active on a subinterval of $\left(t_{0}, t_{f}\right)$ or just at a point. In the former case, the constrained (active) subarc will be characterised by the entry time $t_{l}$ and the exit time $t_{2}$ with $t_{0} \leq t_{1} \leq t_{2} \leq t_{f}$.

The functions appearing in (12)-(19) are assumed to be sufficiently continuously differentiable with respect to their arguments. Note that the definition of $U$ allows discontinuities in controls and thus implies corners (cusps) in the states, so that $\mathrm{X}$ comprises piecewise smooth functions. This is a practical necessity, as many real-world applications of optimal control involve bang-bang type inputs.

Problem (12)-(19) is infinite-dimensional: its solution is not a finite vector of numbers, but a function. For a real-life application it is impossible to guess the optimal function, so a recourse to approximate methods is necessary. They attempt to find a finitedimensional representation of the solution which is accurate at the nodes of the representation, has acceptable error between the nodes and converges to the true function as the number of nodes 
tends to infinity, if second order sufficient conditions hold.

There are two main approaches to the solution of the problem, see e.g. Betts [1],[2]. The direct approach replaces the continuous time interval with a grid of discrete points, thus approximating it with a finite-dimensional problem, albeit of high dimension (hundreds of discretised variables). The indirect approach preserves the infinite-dimensional character of the task and uses the theory of optimal control to solve it.

\section{Direct COLlOCATION APPROACH}

The basic approach for solving optimal control problem by direct collocation approach is to transform the optimal control problem into sequence of nonlinear constrained optimisation problems by discretising of the state and/or control variables, see e.g. Hargraves and Paris [4].

The direct collocation approach is based on the discretisation of both the state and control variables. The following derivation is mainly taken from von Stryk and Bulirsch [6]. The duration time of the optimal trajectory is divided into subinterval as follows:

$t_{0=} t_{1}<t_{2}<t_{3} \ldots \ldots<t_{k}=t_{f}$

The value of the control variables at the centre is given by

$u\left(t_{c, j}\right)=\frac{u\left(t_{j}\right)+u\left(t_{j+1}\right)}{2}$

The piecewise linear interpolation is used to prepare for the possibility of discontinuous solutions in control. The state variable $x(t)$ is approximated by a continuously differentiable and piecewise Hermite-Simpson cubic polynomial between $x\left(t_{j}\right)$ and $x\left(t_{j+1}\right)$ on the interval $t_{j} \leq t \leq$ $t_{j+l}$ of length $q_{j}$ :

$x_{\text {app }(t)}=\sum_{r=0}^{3} c_{r}^{j} \frac{t-t_{j}}{p_{j}}$

$c_{o}^{j}=x\left(t_{j}\right)$

$c_{1}^{j}=q_{j} f_{j}$

$c_{2}^{j}=-3 x\left(t_{j}\right)-2 q_{j} f_{j}+3 x\left(t_{j+1}\right)-q_{j} f_{j+1}$

$c_{3}^{j}=2 x\left(t_{j}\right)+q_{j} f_{j}-2\left(t_{j+1}\right)+q_{j} f_{j+1}$

where:

$$
\begin{aligned}
f_{j}=f\left(x\left(t_{j}\right), u\left(t_{j}\right), t_{j}\right), q j=t_{j+1}-t_{j} \\
t_{j} \leq t \leq t_{j+1}, j=1,2, \ldots, k-1
\end{aligned}
$$

The value of the state variables at the centre point of the cubic approximation

$x_{c, j}=\frac{x\left(t_{j}\right)+x\left(t_{j+1}\right)}{2}+\frac{q\left\lfloor f\left(t_{j+1}\right)\right\rfloor}{8}$

and the derivative is

$\frac{d x_{c, j}}{d t}=\frac{3\left\lfloor x\left(t_{j}\right)+x\left(t_{j+1}\right)\right\rfloor}{2 q}-\frac{q\left\lfloor f\left(t_{j}\right)+f\left(t_{j+1}\right)\right\rfloor}{4}$

In addition, the chosen interpolating polynomial for the state and control variables must satisfy the midpoint conditions for the differential equations as follows:

$$
f\left(x_{a p p}\left(t_{c, j}\right), u_{a p p}\left(t_{c, j}\right), t_{c, j}\right)-\dot{x}_{a p p}\left(t_{c, j}\right)=0
$$

The equations (12)-(19) in Section 4 now can be defined as a discretised problem as follows:

$\min f(\mathrm{Y})$

$$
\begin{aligned}
& f\left(x_{a p p}(t), u_{a p p}(t), t\right)-\dot{x}_{a p p}\left(t_{c, j}\right)=0 \\
& x_{a p p}\left(t_{1}\right)-x_{1}=0 \\
& \Psi\left(x_{a p p}\left(t_{k}\right), t_{k}\right)=0 \\
& C\left(x_{a p p}(t), u_{a p p}(t), t\right) \leq 0 \\
& S\left(X_{a p p}(t), t\right) \leq 0
\end{aligned}
$$

where Xapp, Uapp are the approximation of the state and control, constituting $Y$ in (26).

\section{NUMERICAL RESULTS}

This above discretisation approach has been implemented in the DIRCOL package [5] which employed the sequential quadratic programming method SNOPT by Gill et al. [3]. The initial position is $\{0,0,0,0,15,0\}$ and the final condition is $\{25,0,0,0,15,0\}$. The container mass $m_{c}$ is $47000 \mathrm{~kg}$ and the trolley mass $m_{t}$ is $33000 \mathrm{~kg}$. The virtual hoisting motor mass $G_{h}$ is $67570.7383 \mathrm{~kg}$ and the virtual trolley motor mass $G_{t}$ is $14701.5 \mathrm{~kg}$. The hoisting force is is constrained with maximum value $50000 \mathrm{~N}$ and minimum value $0 \mathrm{~N}$. Furthermore the trolley force is bounded by $10000 \mathrm{~N}$ on the maximum and $-10000 \mathrm{~N}$ on the minimum values. The state constraint on the trolley speed is active and saturated on the maximum an minimum value, see Figure 3.

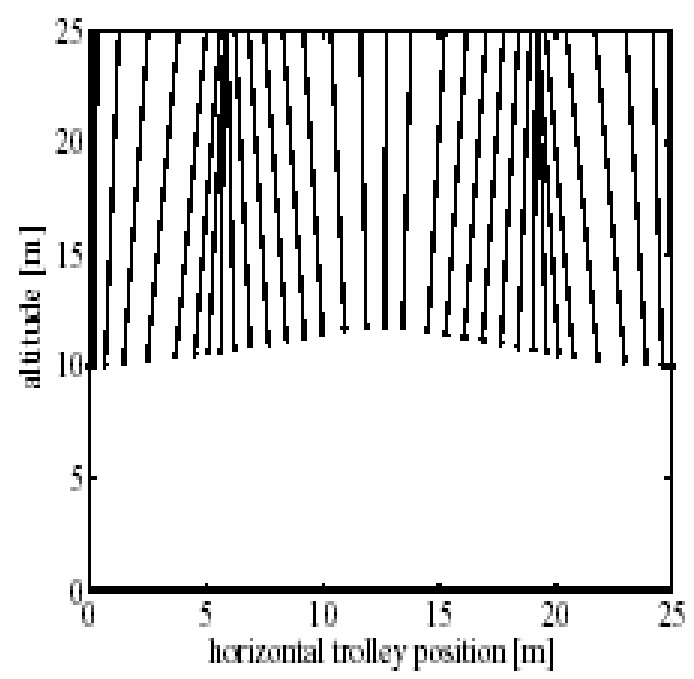

Fig. 2. Altitude vs. horizontal position

\section{CONCLUSION}

In this paper, the direct method approach based on the direct collocation used to solve the minimum-time traversing of the container crane. The main advantage of this approach compare to indirect method is that the need of deriving the necessary conditions and costate equations does not require. The optimal trajectory shows that some state and control constraints are active during the traversing the container. The state constraint on the trolley speed is active on the maximum value, moreover the trolley and hoisting forces are saturated on the optimum values. The most interesting is the singularity on the trolley force which is quite difficult to solve for both direct and indirect method especially for the highly nonlinear optimal control of the traversing container. 


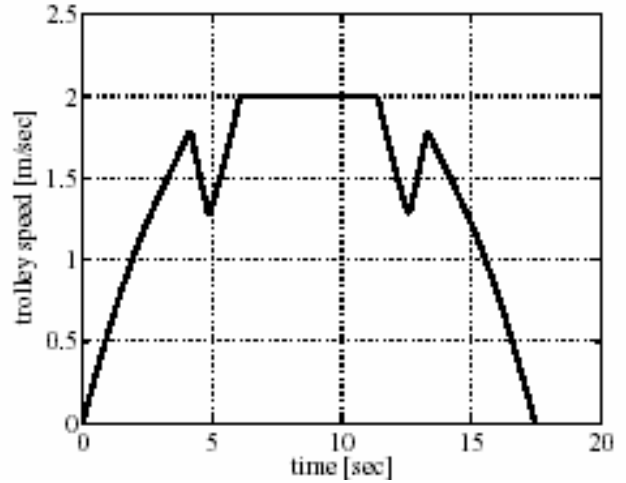

Fig. 3. Trolley speed vs. time

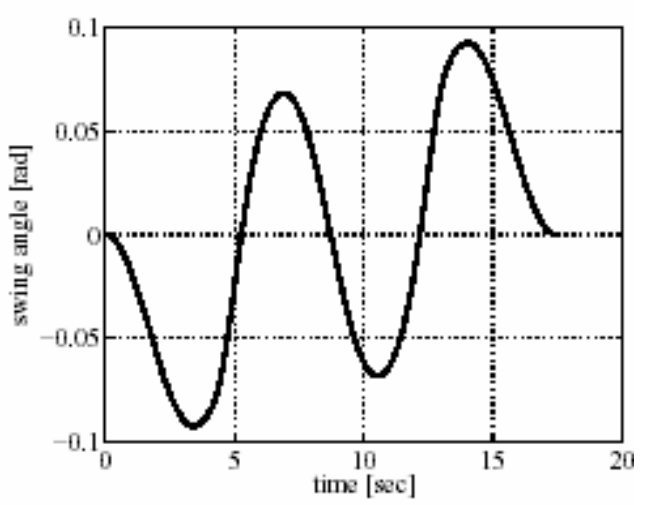

Fig. 4. Swing angle vs. time

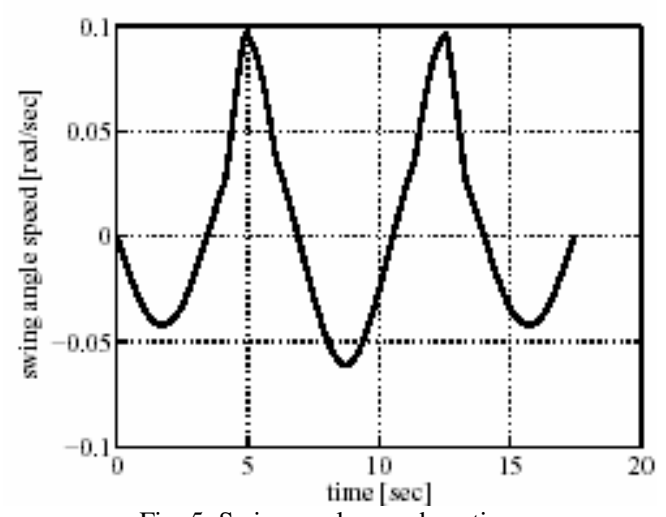

Fig. 5. Swing angle speed vs. time

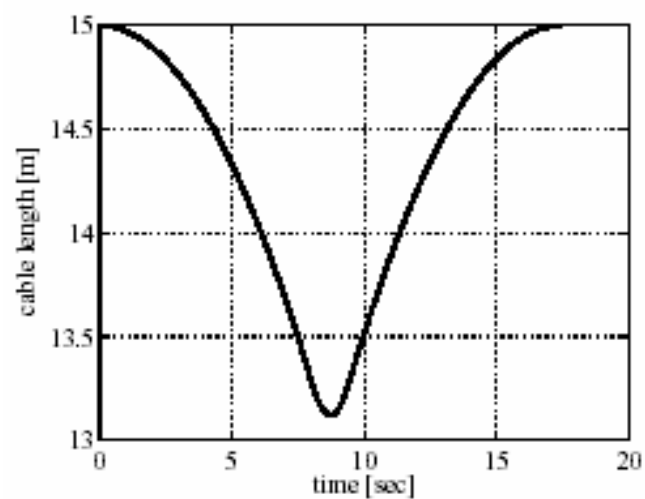

Fig. 6. Cable length vs. time

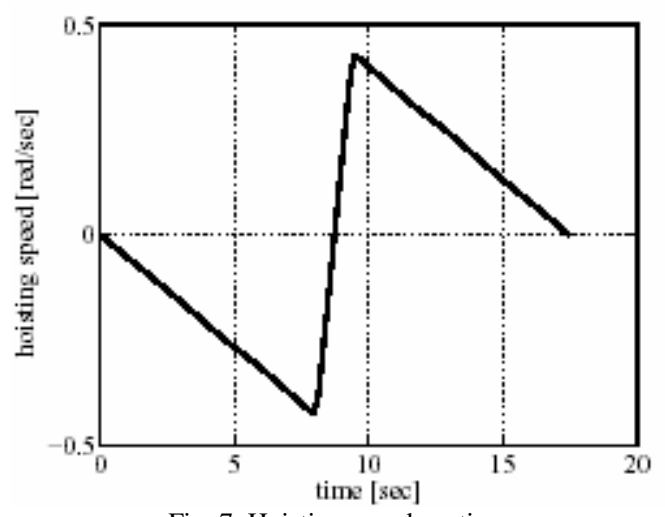

Fig. 7. Hoisting speed vs. time

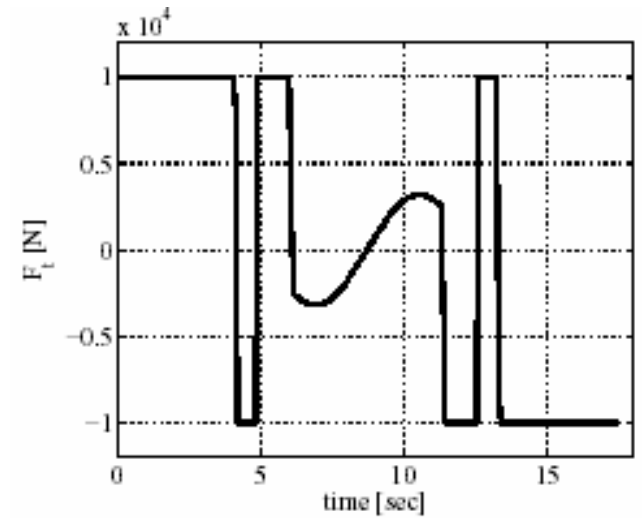

Fig. 8. Trolley force vs. time

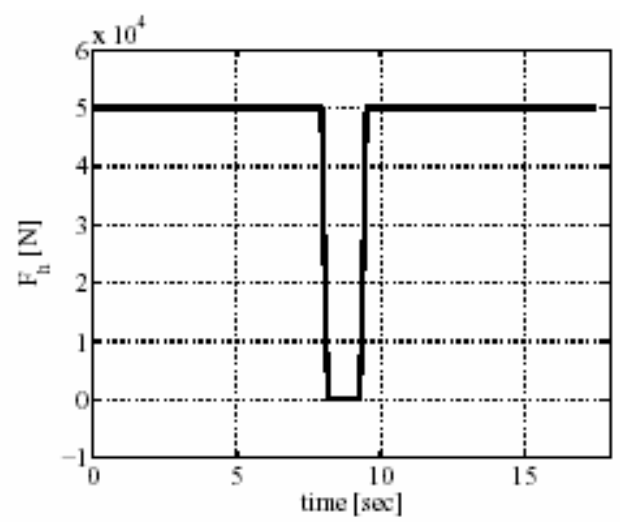

Fig. 9. Hoisting force vs. time

\section{REFERENCES}

1. J. T. Betts., "Survey of numerical methods for trajectory optimization". Journal of Guidance, Control, and Dynamics, 21(2):193-207, 1998.

2. J. T. Betts. Practical Methods for Optimal Control Using Nonlinear Programming. SIAM, Philadelphia, 2001.

3. P. E. Gill, W. Murray, and M. H. Wright., "SNOPT: An SQP algorithm for large scale constrained optimization". SIAM Journal on Optimization, 12(4):979-1006, 2002.

4. C. R. Hargraves and S. W. Paris., "Direct trajectory optimization using nonlinear programming and collocation". Journal Guidance, Control, and Dynamics, 10(4):338- 342, 1987.

5. O. von Stryk., "User's guide for DIRCOL - a direct collocation method for the numerical solution of the optimal control problem". Technische Universität Darmstad. 1999

6. O. von Stryk and R. Bulirsch., "Direct and indirect methods for trajectory optimization". Annals of Operations Research, 37(14):357-373, 1992 